\title{
(Film-) Genres und die Reduktion von Unsicherheit
}

\author{
Volker Gehrau
}

Die Studie gebt dem Zusammenhang zwischen der Verwendung von Genrebezeichnungen und der Reduktion von Unsicherheit nach. Im ersten Teil des Beitrags werden dazu vier theoretische Modellierungen vorgestellt: In der kulturellen Modellierung resultiert die Reduktion von Unsicherbeit aus der über Genres geschaffenen Anschlussfäbigkeit zwischen einem konkreten Angebot und dem Gesamtangebot. Die Anschlussfähigkeit des einzelnen Angebots an bereits erfolgreich vermarktete Angebote sowie die Publikumswünsche steht im Vordergrund der ökonomischen Modellierung. In der psychologischen Modellierung bieten Genrezuordnungen Anschlussmöglichkeit an bereits gemachte kognitive und emotionale Erfabrungen. In der sozialen Modellierung dienen Genrebezeichnungen dazu, mittels Sicherung von Anschlusskommunikation Unsicherheit bei gemeinsamen Medienhandlungen, insbesondere der Kommunikation über Medienangebote, zu reduzieren. Im zweiten Teil der Studie werden die Überlegungen auf das Publikum fokussiert: Demnach müssten Zuschauer in der Kommunikation über Medienangebote verstärkt Genrebezeichnungen benutzen, wenn die Situation unsicher ist. Innerhalb derselben Situation müsste das individuelle Sicherheitsgefübl ansteigen, wenn auf Genrebezeichnungen zurückgegriffen werden kann. Die vorliegenden Daten aus einer mündlichen Befragung sowie einer Reibe von Rezeptionsexperimenten bestätigen die Vermutungen.

Keywords: Film- und Fernsehforschung, Rezeptionsforschung, Genre, Unsicherheit, Steuerungsfunktion, Befragung, Rezeptionsexperiment

\section{Ansatz}

Der nachfolgende Beitrag thematisiert die Frage, welche Funktion die Verwendung von Genrebegriffen zur Bezeichnung von Film- und Fernsehangeboten erfüllt. ${ }^{1}$ Er konzentriert sich dabei auf den Aspekt des Umgangs mit Unsicherheit. Durch die Zuordnung eines Angebots zu einem Genre werden diesem bestimmte Eigenschaften zugeschrieben, andere mit großer Wahrscheinlichkeit erwartet und wieder andere von vornherein ausgeschlossen und damit Unsicherheit im Handeln mit dem entsprechenden Angebot reduziert. Diese Grundidee ist nicht neu; sie wird in einer Vielzahl theoretischer Ansätze zu Genres explizit oder implizit formuliert. Die hier vorgestellte Argumentation basiert sowohl auf einer Diskussion wichtiger theoretischer Ansätze im Feld von Filmund Fernsehgenres in Bezug auf den Punkt Unsicherheitsreduktion als auch auf empirischen Daten.

Den Ausgangspunkt bildet die Überlegung, dass Genrebegriffe eine über Erwartungen konkretisierte Beziehung zwischen Film- und Fernsehangeboten einerseits und dem Publikum andererseits konstituieren. Die Konkretisierung der Erwartungen findet über die Sicherung von Anschlussfähigkeit statt. Die Möglichkeit, nicht nur über das einzelne Angebot zu kommunizieren, sondern über die Gleichartigkeit vieler Angebote,

1 Ich danke Cornelia Spallek für Anmerkungen zu dem Manuskript sowie Iris Morgenstern und Jens Wolling für hilfreiche Hinweise zu einer vorhergehenden Version des Beitrags. 
schafft diese Anschlussfähigkeit, und Genrebegriffe fassen diese Gleichartigkeit in einen leicht kommunizierbaren Begriff.

Die theoretische Argumentation folgt der Einteilung in Angebot und Publikum und differenziert jeweils danach, ob die Reduktion von Unsicherheit mit oder ohne Referenz auf den anderen Bereich stattfindet. Damit lassen sich vier unterschiedliche Felder einteilen, in denen die hier zu untersuchende Reduktion von Unsicherheit im Kern durch (a) kulturelle, (b) ökonomische, (c) psychologische sowie (d) soziale Modellierung erklärt wird. Die kulturelle und die ökonomische Modellierung fokussiert auf das Angebot (letztere mit Bezug zum Publikum), die psychologische und die soziale Modellierung fokussiert auf das Publikum (erstere mit Bezug zum Angebot). Die kulturelle Modellierung von Genrebegriffen stellt eine Verbindung eines einzelnen Angebots zum Gesamtangebot her und reduziert Unsicherheit durch die Schaffung kultureller Anschlussfähigkeit. Die ökonomische Modellierung berücksichtigt zudem die Verbindung zwischen Angebot und Publikum. Sie erklärt die Reduktion von Unsicherheit durch das Bestreben, an ökonomisch erfolgreiche oder Erfolg versprechende Angebote mit einem konkreten Angebot anzuknüpfen. Die psychologische Modellierung stellt demgegenüber eine Verbindung zwischen den vorliegenden emotionalen und kognitiven Rezeptionsphänomenen und der Erinnerung an vorangegangene emotionale und kognitive Rezeptionsphänomene her. Die soziale Modellierung kommt bei der Kommunikation über Medienangebote zum Tragen. Hier wird Unsicherheit über die Sicherung sozialkommunikativer Anschlussfähigkeit vollzogen. Die Unterscheidung zwischen den vier Modellierungen dient der Vereinfachung der Argumentation. De facto lassen sich die vorhandenen theoretischen Ansätze zu Genres nicht eindeutig zuordnen, da sie explizit oder implizit mehrere Modellierungen in ihre jeweilige Argumentation mit einbeziehen. Trotzdem lassen sich einzelne Aspekte der theoretischen Ansätze den vier Modellierungen zuordnen.

Im empirischen Teil des Beitrags werden Befragungsdaten zur Publikumsseite vorgestellt, mit denen der Zusammenhang zwischen Unsicherheit und der Verwendung von Genrebegriffen untersucht wird. Den Ausgangspunkt bildet die Hypothese, dass Genrebegriffe verwendet werden, um Unsicherheit zu begegnen. Deshalb müsste einerseits die Wahrscheinlichkeit der Verwendung von Genrebegriffen größer sein, wenn der Verwendungskontext viel Unsicherheit birgt, als in Verwendungskontexten mit wenig Unsicherheit. Andererseits sollte innerhalb desselben Verwendungskontextes die Unsicherheit abnehmen, wenn Genrebegriffe verwendet werden.

\section{Genres aus unterschiedlicher Perspektive}

\subsection{Genres aus kultureller Perspektive}

Die kulturelle Perspektive geht vom Angebot aus und untersucht in erster Linie Filmgenres. Sie arbeitet Ähnlichkeiten zwischen Filmangeboten heraus und fasst diese zu Segmenten zusammen. Bei ausreichender Ähnlichkeit und Stabilität der Segmente werden Genres konstituiert und mit Genrebezeichnungen benannt. Die Konstitution und Benennung der Genres findet in Anlehnung an den kulturellen Hintergrund z. B. literarische Genres - sowie die gesellschaftlichen Gegebenheiten statt. Die Zuordnung des konkreten Angebots zu einem Genre findet über Ähnlichkeit statt. Wird ein Angebot einem Genre zugeordnet, so ist damit die Erwartung verbunden, dass das konkrete Angebot den typischen Merkmalen des Genres entspricht. Die Reduktion von Unsicherheit kommt in der kulturellen Modellierung von Genres durch die 
Sicherstellung kultureller Anschlussfähigkeit des einzelnen Angebots an andere Angebote zustande.

Beispielhaft für die kulturelle Betrachtungsweise sind Bawdens Ausführungen zu Genres in Buchers Enzyklopädie des Films:

„Genre: Eine Gruppe von fiktionalen Filmen mit gewissen gemeinsamen Merkmalen. Diese gemeinsamen Merkmale können geographischer (beispielsweise Western), zeitlicher (beispielsweise Ritterfilme), motivischer (beispielsweise Musical), dramaturgischer (beispielsweise Epischer Film) oder produktionstechnischer Natur sein (beispielsweise Ausstattungsfilm) - meist ist es eine Kombination von mehreren derartigen Elementen.“ (Bawden 1977: 292)

Die Ansätze, die ich zur kulturellen Perspektive von Genres zähle, stammen hauptsächlich aus den Bereichen Filmwissenschaft, Film- und Genretheorie sowie Filmkritik. Altman (2000: 216-226) unterscheidet hier - in Anlehnung an die Semiotik - semantische und syntaktische Ansätze. Semantische Ansätze konstruieren die Ähnlichkeitsbeziehung zwischen den Filmwerken nach inhaltlichen und filmischen Elementen. Ein Film gehört dann einem Genre an, wenn er dessen typische Elemente aufweist. Krimis könnte man demnach über das Vorkommen von Verbrechern und Polizei konstituieren. Im syntaktischen Ansatz werden Genres nach Beziehungen zwischen Elementen abgegrenzt. So könnte man Krimis über ein Verbrechen abgrenzen, das zu Beginn der Geschichte begangen und im Verlauf der Geschichte aufgeklärt wird. Semantische Ansätze haben nach Altman (2000: 220) den Vorteil, auf die meisten Filme anwendbar zu sein, sie können aber die Entstehung und Logik der Genres nicht erklären; syntaktische Ansätze sind demgegenüber zwar auf weniger Filme anwendbar, können dafür aber die Entstehung und Logik der Genres erklären.

Semantische Ansätze finden sich vornehmlich im Feld der Filmtheorie bzw. Filmkritik. Filme werden nach Genres zugeordnet, um einerseits die Besonderheit des konkreten Filmwerks herauszuarbeiten (Gehrau 2001: 97) und andererseits Parallelen zu anderen aufzuzeigen. Z. B. geht Neal (2000) zum Teil semantisch vor, indem er die Charakteristika der wichtigen Hollywood-Genres herausarbeitet. Tudor (1977: 16-17) kritisiert solches Vorgehen, denn beispielsweise ist der Western ziemlich gut über das Merkmal „Amerika in der Zeit zwischen 1860 und 1900“ abzugrenzen; dieses sagt aber fast nichts über den eigentlichen Charakter von Western aus.

Die meisten genretheoretischen Ansätze folgen eher der syntaktischen Variante. Für T. Sobchack (1977) bildet die Poetik von Aristoteles und deren Ansatz, Geschichten zu erzählen, den Ausgangspunkt heutiger Genrefilme. Die typischen Geschichten und Charaktere seien fast dieselben geblieben, auch wenn sich deren Realisation im Laufe der Zeit und unterschiedlicher Moden veränderte. V. Sobchack (1982) vermutet den Ursprung der gängigen Genrefilme in klassischen Mythen und Ritualen. Die dort festgelegten Handlungsweisen werden in den Geschichten der Genrefilme lediglich variiert. McConnell (1977) sieht das Genre als eine bestimmte Art an, einen Film zu realisieren, die sich an bereits bestehende Filmwerke anlehnt.

In beiden Varianten findet durch die Zuordnung eines Films zu einem Genre eine Reduktion von Unsicherheit statt, denn der zugeordnete Film erbt zumindest einige semantische und/oder syntaktische Eigenschaften des Genres. Dieses Wissen generiert Erwartungen an den konkreten Film und erleichtert es, ihn zu analysieren und zu kritisieren. 


\subsection{Genres aus ökonomischer Perspektive}

Auch die ökonomische Perspektive setzt beim Angebot an. Sie thematisiert die Frage, inwiefern die ökonomischen Strukturen bei der Produktion und Vermarktung von Filmen die Entwicklung von Genres beeinflusst haben. Dabei bezog sich die Argumentation anfangs auf die Strukturen der Filmindustrie in Hollywood, später auf die Entwicklung der Film- und Fernsehproduktion allgemein. Auch in dieser Perspektive handelt es sich bei Genres um Gruppen von Filmen mit bestimmten Merkmalen. Durch den Bezug auf Genres wird Unsicherheit bei Produktion und Absatz der Produkte reduziert. Das geschieht zum einen über die Schaffung von Standards und zum anderen über die Möglichkeit, an erfolgreiche Produkte anzuschließen.

Eine gewisse Standardisierung ergibt sich beim Herstellen von Filmen fast zwangsläufig aus ökonomischen Zwängen, denen durch Minimierung der Produktionskosten Rechnung getragen wird. Das führt zu der Idee, Requisiten, Kulissen, Kostüme etc. mehrfach zu benutzen. (Altman 2000: 184-187) Ähnliches vollzog sich, als die Filmindustrie versuchte, die Filmproduktion durch Orientierung an den Publikumserwartungen zu standardisieren. Man lehnte sich bei der Produktion neuer Filme an gemeinsame Merkmale bereits erfolgreich vermarkteter Filme an. Zudem bildeten sich aus dem Erfolg oder Misserfolg vorhandener Filme Erwartungen darüber, was das Publikum zu sehen wünscht. Um den Erfolg eines Filmes kalkulieren und maximieren zu können, orientiert sich die Produktion neuer Filme an diesem Wissen. Schweinitz (1994) sieht in diesem Phänomen den Beginn des systematischen Operierens mit Genres und charakterisiert die damalige Situation so:

„Zur Jahresmitte 1911 konnte man von einer großen Filmgesellschaft regelmäßige Lieferungen, bestehend aus einem Melodrama, einem Western und einer Comedy, erwarten“. (Schweinitz 1994: 100)

Hinzu kommt die Vermarktung audio-visueller Produkte. Hierbei spielt weniger die Orientierung an Genre-Standards eine Rolle als die Verwendung von Genrebegriffen zur Ankündigung und Verbreitung des Produkts. Gehrau (1999: 78-80) vergleicht das Vorgehen mit der Verpackung eines Produktes, die beim Kunden Interesse und Erwartungen wecken sollen. Die Genrebezeichnung ebnet den Weg eines bestimmten Produktes in Richtung Zielgruppe und macht diese auf das Produkt aufmerksam. Altman (2000: 100-122) kann zeigen, dass dieses Vorgehen in den frühen Jahren der Filmvermarktung einen entscheidenden Beitrag zur Genese von Genrebezeichnungen leistete. Studios replizierten erfolgreiche Filmkonzepte, so dass Reihen entstanden, an denen die Studios die Rechte besaßen. Andere Studios realisierten ähnliche Produkte, um am Erfolg zu partizipieren. Sie durften aber die Titel nicht verwenden. So umschrieben sie erst die Charakteristika der erfolgreichen Reihen mit Adjektiven, aus denen sich später oft Genrenamen entwickelten.

Aus der ökonomischen Perspektive hat der Zwang, Unsicherheit zu reduzieren - im Sinne der Reduktion ökonomischer Risiken -, sowohl zur Genese von Genrebegriffen als auch zur Orientierung an Genrevorgaben geführt. Die Unsicherheitsreduktion ergibt sich einerseits aus der Anschlussfähigkeit an ökonomisch erfolgreiche Produkte und andererseits aus der Anschlussfähigkeit an Publikumserwartungen. 


\subsection{Genres aus psychologischer Perspektive}

Aus der psychologischen Perspektive reduzieren Genres Unsicherheit durch Anschlussfähigkeit an bereits gemachte kognitive und emotionale Erfahrungen. Genres organisieren die kognitiven und emotionalen Erfahrungen als spezielles Wissen (für Film- und Fernsehangebote), mit dessen Hilfe spezielle Erwartungen gebildet werden. Psychologisch wirksam werden die Genres durch ein Zusammenspiel von Bottom-up- und Topdown-Prozessen. Bottom-up aktivieren Wahrnehmungen bereits gemachte kognitive und emotionale Genreerfahrungen, die wiederum top-down Erwartungen aktivieren und die kognitive und emotionale Verarbeitung steuern. Bei den psychologischen Ansätzen zu Genres lassen sich kognitive und emotionale Ansätze unterscheiden.

Es dominieren die kognitiven Ansätze, die sich im Kern implizit oder explizit auf die kognitive Schematheorie beziehen. Die moderne Variante der kognitiven Schematheorie wurde durch drei ähnliche Konzepte eingeführt: Frames von Minsky (1975), Scripts von Schank und Abelson (1977) sowie Schemata von Rumelhart (1975). Nach Rumelhart sind Schemata komplexe Wissensstrukturen, die aus einem Geflecht von Variablen bestehen. Ein Schema wird durch bestimmte Reize aktiviert. Bei der Aktivierung belegt die aktivierende Reizkonstellation einige Variablen des Schemas, die wiederum die Belegung anderer Variablen des Schemas beeinflussen. Im Modell Rumelharts setzt Aktivierung eines Schemas die Variablen nicht auf einen festgelegten Wert, sondern der Wertebereich möglicher Belegungen wird eingeschränkt und bestimmte Ausprägungen werden wahrscheinlicher als andere. Nach der Idee des Wahrnehmungszyklus 'von Neisser (1979) aktivieren bestimmte Wahrnehmungen Schemata, die dann die weitere Wahrnehmung leiten. Dadurch wird eine ökonomische Informationsverarbeitung sichergestellt. Es muss lediglich anhand wichtiger Bestandteile des Schemas geprüft werden, ob das Schema auf die folgenden Wahrnehmungen passt. Danach kann sich die Wahrnehmung auf untypische Merkmale konzentrieren, die über das Schema hinausgehende Zusatzinformationen liefern (Waldmann 1990: 54-60). Das Schema leitet die weitere Wahrnehmung nicht nur; es generiert auch Erwartungen an die folgenden Wahrnehmungen. Mit den Erwartungen entsteht die Möglichkeit, Schemata bewusst zu verändern (Baumgartner/Trauner 1996: 166-167).

Filmverstehen ist laut Bordwell (1992) ein kognitiver Konstruktionsprozess, bei dem die Zuschauer, vom Filmmaterial ausgehend und auf Vorwissen zurückgreifend, provisorische Hypothesen über den Filmverlauf bilden. Dabei orientieren sich die Zuschauer einerseits an Handlungsschemata bezüglich der Narration und der Situation und andererseits an Figurenschemata über Rollen und Personen sowie deren Motive und Ziele. Die Vorhersehbarkeit ist - laut Bordwell - bei der Filmrezeption besonders wichtig, da man im Gegensatz zur Textlektüre nicht zurückblättern kann. Das narrative FormInhalt-Korrespondenzgitter steht im Zentrum des Ansatzes von Ohler (1994). Er beschreibt die Filmverarbeitung mit Hilfe eines kognitiven Prozessmodells, bei dem Reize aus dem sensorischen Kurzzeitspeicher und Informationen aus unterschiedlichen Wissensbeständen des Langzeitgedächtnisses im zentralen Prozessor zu einem Situationsmodell integriert werden. Dieses stellt eine Vereinfachung der eingehenden Reizkonstellation dar, da der zentrale Prozessor nur über eine begrenzte Kapazität verfügt. Ohler (1994: 32-41) unterscheidet drei Wissensbasen, auf die der zentrale Prozessor bei der Filmverarbeitung zurückgreift: (1) das generelle Weltwissen über persönliche und kulturell tradierte Alltagserfahrungen, (2) narratives Wissen über genretypische Personen, Konstellationen, Handlungen und Plots sowie (3) das Wissen über filmische Darstellungsformen. Unter anderem untersuchte Ohler den Zusammenhang zwischen Gen- 
reschemata und Filmerinnerung. Erstens erhöht das Genrewissen die Wahrscheinlichkeit der Erinnerung solcher Szenen, die innerhalb des Genres die Geschichte konstituieren. Zweitens werden solche Szenen nicht erinnert, die durch das Genreschema in den Hintergrund treten, da sie entweder für den Handlungsverlauf irrelevant oder vom Schema selbst vollständig repräsentiert sind. (Ohler 1994: 239-251) Zudem konnte er anhand eines kurzen Ausschnitts belegen, dass Zuschauer aus diesem genrespezifische Annahmen über den Fortgang der Handlung generieren, die im Groben den typischen Handlungsverläufen des Genres entsprechen. (Ohler 1994: 230-238) Schwan (1995) betrachtet Genres als kognitive Schemata, die Informationen über typische Personen, Objekte und Ereignisverläufe in Filmen enthalten, die, wenn sie beim Zuschauer aktiviert sind, die Art seiner Informationsverarbeitung und seiner Filminterpretation steuern: Die Aktivierung eines bestimmten Genres vereinheitlicht die Einschätzung, welche Szenen für den Film wichtig und welche eher unwichtig sind. (Schwan 1995: 32-37) Gehrau (2001) kann zeigen, dass bei geeigneten Szenen bereits binnen einer Sekunde eine relativ sichere Genreidentifikation stattfindet, die insbesondere von der Erkennung typischer Personen sowie auffälliger Schlüsselreize abhängt. (Gehrau 2001: 236-251)

Im Vergleich zur kognitiven bildet die emotionale Modellierung von Genres eher die Ausnahme. Sie betrachtet bestimmte emotionale Konstellationen als typisch für bestimmte Genres. Treten diese Konstellationen auf, wird das Genre (-Gefühl) aktiviert und leitet die weitere Rezeption. Den Ausgangspunkt bilden Emotionstheorien, die entweder körperliche Erregung als Emotion betrachten oder die Bewertung dieser Erregung (Grodal 2000: 4). Nach Scherer (1998) entstehen die Emotionen bei der Rezeption audio-visueller Medienangebote zum einen als Emotionen durch die medieninduzierte Erregung, die im Kontext des Medieninhalts bewertet wird, und zum anderen als Komotion, also der Übernahme der Emotionen der dargestellten Medienfiguren.

Grodal (2000) ist der Erste, der Genres vornehmlich auf emotionaler Basis argumentiert. Seine Ausgangshypothese lautet:

»My hypothesis is that the main genre-formulars and moods of fictive entertainment are often constructed to produce certain emotions, by allowing the viewer to stimulate one from a set of fundamental emotions linked to basic human situations. Mass fiction, in particular, is preduced, consumed and distributed in certain categories, and it seems intuitivly evident that one of the pertinent features distinguish these categories is a set of affect-preducing patterns (such as horror, romance and comedy) [...].«(Grodal 2000: 161)

Grodal unterscheidet drei Dimensionen, welche die Art der emotionalen Rezeption bestimmen und die wichtigen Genres unterscheiden: (a) die Kontextualisierung der Rezeption in Bezug auf die Frage, ob die reale Rezeptionssituation bewusst bleibt oder ausgeblendet wird, (b) die Art der kognitiven und emotionalen Beziehung zu den Medienfiguren sowie (c) die Struktur der Narration, vor allem in Bezug auf die Frage, ob den Figuren das Geschehen eher passiv widerfährt, oder ob die Handelnden es eher aktiv gestalten. Während der Rezeption führt die Kombination der drei Dimensionen zu einer dominanten emotionalen Tönung (einer Art Genregefühl), welche die einzelnen emotionalen Reaktionen steuert. So kann dieselbe Szene, in der z. B. ein Mann einen anderen Mann schlägt, je nach Genrekontext Freude verursachen, weil es lustig ist, Trauer, weil es dramatisch ist, oder Angst, da es spannend ist. (Grodal 2000: 157-164)

Psychologisch betrachtet reduzieren Genres Unsicherheit durch die Anschlussfähigkeit an bereits vorhandene kognitive und emotionale Erfahrungen. Sie generieren Er- 
wartungen, die die kognitive Interpretation, die emotionale Verarbeitung sowie die motivierte Auswahl von Medieninhalten steuern.

\subsection{Genres aus sozialer Perspektive}

Aus sozialer Perspektive dienen Genrebegriffe dazu, Unsicherheit in Kommunikationssituationen zu reduzieren. Sie werden in der Hoffnung benutzt, dem Kommunikationspartner deutlich machen zu können, worüber man redet, um so die sozial-kommunikative Anschlussfähigkeit zu sichern.

Die Argumentation schließt an Luhmanns (1987) Theorie Sozialer Systeme an. Luhmann bezeichnet all jene Reize oder Ereignisse als Information, die im System Komplexität reduzieren, indem sie auf nur eine begrenzte Zahl von Möglichkeiten verweisen (1987: 102-105). Da ständig Informationen auftreten, kann es sein, dass bestimmte Informationen immer in derselben Konstellation oder Reihenfolge auftreten. Diese werden zu Generalisierungen zusammengefasst und durch einzelne Symbole repräsentiert. Die Symbole lassen sich kommunizieren, so dass die Generalisierungen durch Kommunikation sozial abgestimmt werden.

„Symbolische Generalisierungen verdichten die Verweisungsstruktur jeden Sinns zu Erwartungen, die anzeigen, was eine gegebene Sinnlage in Aussicht stellt. Und ebenso gilt das Umgekehrte: Die in konkreten Situationen benötigten [...] Erwartungen führen und korrigieren die Generalisierungen." (Luhmann 1987: 139)

Die konstruktivistische Mediengattungstheorie knüpft daran an und fundiert ein Gattungskonzept über Medienhandlungen. Rusch (1987) greift die Idee der autopoietischen Systeme auf. Lebende Systeme entwickeln sich in Koevolution mit anderen lebenden Systemen, die sich gegenseitig wahrnehmen und miteinander kommunizieren. Durch gemeinsames Handeln in Bezug auf bestimmte Objekte entstehen Konventionen. Zu diesen zählt Rusch auch Begriffe für Mediengattungen. Sie bilden sich im gemeinsamen Operieren von Personen mit Medienangeboten. Die Begrifflichkeit variiert je nach Personen, die sie verwenden, Situationen, in denen sie verwendet werden und Medienangeboten, auf die sie sich beziehen. (Rusch 1987: 230-252) Nach Schmidt (1987) steuern Mediengattungen Handlungen und Erwartungen individuell und sozial. Inhalt und Verlauf von Medienangeboten werden zum Teil vorhersehbar und das individuelle Medienhandeln darauf abgestimmt. Zudem können sich die Handlungen an einem sozialen Referenzsystem orientieren und so eine Verbindung zum sozialen System schaffen. (Schmidt 1987: 166-185) Haben sich bestimmte Erwartungen im sozialen System durchgesetzt, wirkt die Orientierung am und die Verbindung zum sozialen System so stark, dass Gattungsbegriffe und damit verbundene Erwartungen an Inhalt und Aufbereitung auf die Medienproduzenten einwirken und sogar die Organisation von Medienunternehmen bis hin zu Teilen des Mediensystems beeinflussen. (Rusch 1987: 263-269)

Gehrau (2001) geht davon aus, dass Genrebegriffe des Publikums eine entscheidende Rolle bei Gesprächen über audiovisuelle Medienangebote spielen. Sie erleichtern das Verständnis zwischen den Kommunikationspartnern. Durch den Verweis auf das Genre werden individuelle und kulturelle Wissensbestände aktiviert, die es ermöglichen, einen ausreichenden Eindruck des Medienangebots zu erlangen, ohne es konkret kennen zu müssen. Diese Funktion der Verwendung von Genrebezeichnungen tritt aber nicht nur in Alltagsgesprächen auf, sondern auch bei der empirischen Untersuchung von 
Genres mittels Befragung, denn diese schafft eine soziale Kommunikationssituation. (Gehrau 2001: 27-30)

Aus der sozialen Perspektive reduzieren Genres Unsicherheit, indem sie mittels Genrebezeichnungen sowohl die Anschlussfähigkeit der individuellen Erfahrungen sicherstellen als auch einen Bezug zu kulturellen Konventionen herstellen.

\section{Hypothesen und Begriffe}

Die generelle Fragestellung der Studie lautet: Sind Genres und Genrebezeichnungen geeignet, Unsicherheit zu reduzieren? Für die kulturelle und die ökonomische Perspektive bleibt es bei der vorab dargestellten theoretischen Argumentation, zumal beide bereits in vielen Ansätzen erörtert wurden. Empirisch untersucht werden die psychologische sowie die soziale Perspektive mit folgenden Hypothesen:

Soziale Perspektive: Fernsehzuschauer greifen bei der Kommunikation über fiktionale Fernsehangebote immer dann vermehrt auf Genrebegriffe zurück, wenn die Situation Unsicherheit birgt.

Psychologische Perspektive: In einer unsicheren Situation steigt das individuelle Gefühl von Sicherheit an, wenn die Zuschauer auf bekannte Genrevorgaben und -begriffe zurückgreifen können.

Die Studie untersucht die Funktion von Genres am Beispiel der Verwendung von Genrebezeichnungen. Anhand von Kommunikation über Film- und Fernsehangebote lässt sich die Funktion der Genrebezeichnungen nachvollziehen. Bei der Kommunikation über Film- und Fernsehangebote konkurrieren Genrebezeichnungen mit anderen Begriffen, die Medienangebote beschreiben.

Das Angebot, auf das sich die Analyse bezieht, umfasst das fiktionale Fernsehprogramm. Bei der Kommunikation über solche Medienangebote werden unterschiedliche Arten von Begriffen benutzt. Diese lassen sich in die folgenden Begriffsfelder einteilen: (a) Titel, (b) Bewertung, (c) Genre, (d) Personen, (e) Inhalt, (f) Technik, (g) Gattung, (h) Sender etc. (vgl. Gehrau 2001). So könnte ein Fernsehangebot beispielsweise folgendermaßen bezeichnet werden: (a) Lolita, ein (b) fesselndes (c) Gesellschaftsdrama mit (d) Peter Sellers über (e) einen älteren Mann, der sich in ein junges Mädchen verliebt, als (f) schwarz-weiß (g) Film auf (h) arte ausgestrahlt.

Die Angaben Titel, Sender und Inhalt rekurrieren auf das konkrete Angebot. Demgegenüber spezifizieren die Angaben zu Personen und Technik das Angebot unter Bezugnahme auf andere film- und fernsehspezifische Angebote. Genre- und Gattungsbezeichnungen sind Generalisierungen zur Beschreibung von Form und Inhalt von Medienangeboten. Dabei bezeichnet die Gattung die Form bzw. das Format eines Angebots, das Genre hingegen den Inhalt bzw. Gegenstand des Angebots. Im Fernsehangebot sind nach dem Format Gattungen wie Magazine, Shows, Nachrichten etc. zu unterscheiden, im fiktionalen Fernsehangebot im Wesentlichen Filme und Serien. Genrebegriffe sind auf das fiktionale Angebot beschränkt und unterscheiden Filme und Serien nach ähnlichen Inhalten, z. B. in Western, Science Fiction, Drama, Krimi etc.. Insofern korrespondiert das Genre eines fiktionalen Angebotes mit dem Thema eines nicht fiktionalen Angebots, z. B. Sport, Gesundheit oder Politik als Gegenstand eines Magazins. (Gehrau 2001: 108-110) 


\section{Anlage der Teiluntersuchungen}

Die Hypothesen werden anhand von Daten aus zwei empirischen Studien geprüft, einer Befragung und einer Reihe von Rezeptionsstudien, mit denen primär die Bezeichnung und Klassifikation von Fernsehprogrammen untersucht werden sollte ${ }^{2}$.

Die Befragung fand mit einer nach Alter und Geschlecht quotierten Stichprobe der Berliner Wohnbevölkerung statt $(\mathrm{n}=265)^{3}$. Es handelt sich um eine mündliche, standardisierte Befragung, durchgeführt von knapp 60 geschulten studentischen Interviewern. Im Fragebogen wurde unter anderem offen gefragt, was die Befragten gestern und vorgestern nach 20 Uhr im Fernsehen gesehen hatten. Damit sollte eine Gesprächssituation simuliert werden, in der sich zwei Personen über das Fernsehprogramm unterhalten. Die Interviewer hatten die Anweisung, die Antwort wörtlich zu protokollieren. Diese Protokolle wurden daraufhin kodiert, welche Art von Elementen die Befragten benutzen, um die von ihnen gesehenen Sendungen zu bezeichnen. Die Kodierung umfasst das Vorkommen von: Titel („Spiel mir das Lied vom Tod“, „Krieg der Sterne“ etc.), Gattung $^{4}$ (Film, Spielfilm etc.), Genre (Western, Krimi etc.), Sender (ARD, RTL etc.), Inhalt (Kampf, Verfolgung etc.), Personen (Schwarzenegger, Asterix etc.), Bewertung (gut, faszinierend etc.) sowie Produktionsangaben (Zeichentrick, schwarz-weiß etc.).

Die Rezeptionsstudien fanden mit insgesamt 124 studentischen Probanden ${ }^{5}$ statt. Diese sahen mehrere ca. eine Sekunde lange Ausschnitte vornehmlich aus dem fiktionalen Fernsehangebot. ${ }^{6}$ Alle Ausschnitte wurden Probandengruppen vorgeführt und die Teilnehmer direkt im Anschluss an jeden Ausschnitt gebeten, auf einem Fragebogen offen anzugeben, aus was für einem Film bzw. einer Sendung der Ausschnitt ihrer Meinung nach stamme. Die Probanden wurden gebeten, so zu antworten, als hätte sie ein Bekannter gefragt, was gerade im Fernsehen läuft. Die Angaben wurden nach demselben System kodiert wie in der Befragung. Obgleich die Hypothesen lediglich Aussagen über die Genrebezeichnungen machen, werden bei allen Untersuchungsschritten auch die andere Angaben ausgewiesen, um die Effekte bei den Genreangaben an den Effekten der anderen Angaben relativieren zu können.

2 Die hier vorzustellenden Ergebnisse haben insofern den Charakter einer weiter führenden Sekundäranalyse. Für die primäre Fragestellung siehe Gehrau 2001.

3 Obgleich nur knapp 90 Prozent der anvisierten Stichprobe von 300 Interviews realisiert werden konnten, weicht keine Gruppe der acht vorgegebenen Merkmalskombinationen aus Geschlecht und Altersgruppe um mehr als 1,6 Prozentpunkte von den Vorgaben - basierend auf dem Statistischen-Jahrbuch - ab. In der Stichprobe sind Personen allen Alters - von 16 bis 96 Jahren - wie auch aller Bildungsgruppen vertreten, obwohl zu den Letztgenannten keine Vorgaben gemacht wurden.

4 Bei der Kodierung in der Originalstudie wurden alle Angaben über das Format von Fernsehangeboten als Gattung erfasst. Da die hier vorgestellte Auswertung auf das fiktionale Fernsehangebot fokussiert, bestehen die Gattungsangaben fast ausschließlich aus der Nennung Film sowie Synonymen.

5 Die Probanden waren zwischen 18 und 30 Jahre alt, mit einem Durchschnittsalter von 21,7 Jahren $($ Median $=21)$ und befanden sich mehrheitlich im Grundstudium. Knapp zwei Drittel von ihnen $(62 \%)$ waren weiblich.

6 Eine Liste der bei den Rezeptionsexperimenten verwendeten Ausschnitte findet sich im Anhang zum Beitrag. 


\section{Ergebnisse}

\subsection{Genrebezeichnungen in Kommunikationssituationen}

Die Befragten haben unterschiedliche Elemente benutzt, um die von ihnen gesehenen fiktionalen Fernsehangebote zu bezeichnen. In zwei Drittel aller Bezeichnungen einer Fiktionsendung finden sich Titelangaben, in knapp der Hälfte Gattungsangaben. In jeweils gut einem Viertel der Bezeichnungen kommen Verweise auf das Genre oder den Sender vor, in gut einem Fünftel auf den Inhalt und in knapp einem Fünftel auf Personen. Ein Achtel der Bezeichnungen enthält persönliche Bewertungen der Sendung, ein Zwölftel Verweise auf die Produktionstechnik. Die Angabe des Titels eines fiktionalen Fernsehangebots ist das wichtigste Merkmal, um das Angebot im Gespräch mit anderen zu bezeichnen. Obgleich der Titel theoretisch ausreicht, um einen Film zu identifizieren, wird er in den meisten Fällen (53 \%) durch mindestens ein weiteres Bezeichnungselement konkretisiert, zumeist handelt es sich um Angaben über die Gattung, den ausstrahlenden Sender oder das Genre.

Tabelle 1: Bezeichnungselemente für Fiktionangebote (nach Titelangaben)

\begin{tabular}{lccc}
\hline Bezeichnungselement & Vorkommen & \multicolumn{2}{c}{ Davon } \\
\hline Titel & $66 \%$ & ohne Titel & mit Titel* \\
Gattung & $44 \%$ & $80 \%$ & $25 \%$ \\
Genre & $27 \%$ & $50 \%$ & $15 \%$ \\
Sender & $26 \%$ & $35 \%$ & $25 \%$ \\
Inhalt & $21 \%$ & $40 \%$ & $10 \%$ \\
Personen & $18 \%$ & $30 \%$ & $10 \%$ \\
Bewertung & $12 \%$ & $10 \%$ & $15 \%$ \\
Produktion & $7 \%$ & $10 \%$ & $5 \%$ \\
\hline Basis & $\mathrm{n}=194$ & $\mathrm{n}=67$ & $\mathrm{n}=127$ \\
\hline
\end{tabular}

* Die Werte sind - wegen der geringen Fallzahl - auf 5 Prozent gerundet.

Vordergründig erscheinen die Zusatzangaben überflüssig. In der simulierten Gesprächssituation kann der Befragte allerdings nicht sicher sein, dass dem Interviewer der Titel bekannt ist. Mit der Ergänzung könnten die Befragten der Unsicherheit begegnen wollen, vom Interviewer nicht adäquat verstanden zu werden. Interessanter sind allerdings diejenigen Angaben, in denen die Befragten nicht den Titel nennen. Einerseits könnten sie etwas gesehen haben, von dem sie den Titel nicht wissen, oder aber sie wissen ihn, nennen ihn aber nicht, da sie meinen, der Interviewer kenne den Titel wahrscheinlich nicht. In beiden Fällen besteht beim Befragten Unsicherheit, im ersten Fall darüber, was er gesehen hat, im zweiten Falle darüber, wie die Kommunikation am besten gelingt. Wenn der Titel nicht genannt wird, greifen die Befragten in acht von zehn Fällen auf die Gattung zur Bezeichnung zurück, in jedem zweiten Fall auf das Genre. Auch andere Angaben werden gemacht, wenngleich deutlich seltener.

Demnach werden Genreangaben in alltäglichen Gesprächen über fiktionale Fernsehangebote benutzt, und zwar vor allem dann, wenn das Angebot nicht über den Titel identifiziert wird. Gemäß der ersten Hypothese können beide Resultate als Indiz dafür gewertet werden, dass Genreangaben den Gesprächspartnern beim Reden über Fernsehangebote dazu dienen, die Unsicherheit über das Gelingen der Kommunikation zu reduzieren. 


\subsection{Genrebezeichnungen bei der Erkennung von Ausschnitten}

Auch bei den Rezeptionsstudien handelt es sich um Befragungen, die der Kommunikationssituation geschuldete Unsicherheit tritt also auch in dieser Untersuchung auf. Hier haben die Probanden aber keinen Film gesehen, sondern lediglich einen Ausschnitt. In Bezug auf die Unsicherheit unterscheiden sich die Situationen insofern, als zur Unsicherheit über das Gelingen der Kommunikation die Unsicherheit darüber hinzukommt, aus welchem Film der Ausschnitt stammen könnte. Bei der Erkennung und Bezeichnung von Fiktion-Ausschnitten sind vor allem Genrebegriffe wichtig; die Probanden benutzten sie in fast drei Viertel aller Fälle. Gut die Hälfte der Bezeichnungen enthält Gattungsangaben. Mittlere Bedeutung haben - mit ca. einem Fünftel Vorkommen - die Inhaltsangaben und Titelnennungen, gut jede sechste Angabe umfasst Personennennungen, knapp jede achte Produktionshinweise. Lediglich bei der Bezeichnung jeder zwanzigsten Rezeption nehmen die Probanden Bewertungen vor; auf Sender wird praktisch nie verwiesen.

Genres - eingeschränkt auch Gattungen - sind als klassifizierende Angaben bei der Einordnung und Bezeichnung kurzer Filmausschnitte bedeutsamer als bei der bloßen Bezeichnung gesehener Filme, wohingegen konkrete Angaben wie Titel oder Sender bei der Einordnung an Bedeutung verlieren.

Tabelle 2: Bezeichnungselemente bei Ausschnitterkennung (Differenzen zur Befragung)

\begin{tabular}{lccc}
\hline Bezeichnungselement & Vorkommen & $\begin{array}{c}\text { Differenz zur } \\
\text { Gesamtbefragung }\end{array}$ & $\begin{array}{c}\text { Differenz zur Gruppe } \\
\text { Abitur/Studium } \\
\text { unter 30 Jahren* }\end{array}$ \\
\hline Genre & $72 \%$ & 45 & 50 \\
Gattung & $51 \%$ & 7 & 10 \\
Inhalt & $22 \%$ & 1 & 0 \\
Titel & $19 \%$ & -47 & -50 \\
Personen & $17 \%$ & -1 & -5 \\
Produktion & $12 \%$ & 5 & 0 \\
Bewertung & $5 \%$ & -7 & -5 \\
Sender & $0 \%$ & -26 & -20 \\
\hline Basis & $\mathrm{n}=779$ & & \\
\hline
\end{tabular}

*Die Werte sind - wegen der geringen Fallzahl - auf 5 Prozent gerundet.

In Tabelle 2 sind die Differenzen in Prozentpunkten zwischen dem Gesamtergebnis der Rezeptionsstudien und dem Gesamtergebnis der Befragung einerseits sowie der entsprechenden Alters- und Bildungsgruppe andererseits wiedergegeben. Streng genommen ist nur die Differenz zwischen den vergleichbaren Gruppen interpretierbar. Die Vergleichsgruppe ist insoweit problematisch, als sie auf lediglich 51 Fällen basiert. Das Problem kommt aber praktisch nicht zum Tragen, da die Unterschiede jeweils zwischen den Bezeichnungselementen deutlich größer ausfallen als die Unterschiede zwischen den jeweiligen Gruppendifferenzen.

Die Ähnlichkeiten des Vorkommens einiger Elemente zwischen der Befragung und der Rezeptionsstudie sind fast verwunderlicher als die Unterschiede. Die Wahrscheinlichkeit, dass bei der Bezeichnung auf den Inhalt oder auf beteiligte Personen verwiesen 
wird, ist bei beiden nahezu identisch. Produktionsangaben sind beim Einordnen von Ausschnitten etwas wahrscheinlicher. Größer fallen die Differenzen bei den Gattungsangaben und Bewertungen aus. Es ist aber schwierig zu beurteilen, wie aussagekräftig die Differenzen - von ca. 7 Prozentpunkten - sind, da der Probandenauswahl der Rezeptionsstudien kein Zufallsverfahren zugrunde liegt und die Befragung auf einer Quotenstichprobe basiert, womit die Voraussetzung für die üblichen Testverfahren fehlt. Ignoriert man die methodischen Einwände und testet die Differenzen nach $t$, so liegen deren Signifikanzen um $p=0,05$. Die Differenzen können also nicht sicher als tatsächlich vorhanden interpretiert werden - im Gegensatz zu den großen Differenzen von fast 50 Prozentpunkten Anstieg bei den Genreangaben und Abnahme bei den Titelangaben sowie den ca. 25 Prozentpunkten Abnahme bei den Senderangaben. Diese Differenzen sind nach t eindeutig signifikant, trotz Anwendung der konservativsten Varianzschätzung für die Prozentwertdifferenzen. ${ }^{7}$

Die Tatsache, dass bei der Erkennung von Ausschnitten weniger Titel- und Senderangaben gemacht werden, ist auf das Problem zurückzuführen, den Originalfilm oder -sender zu erkennen. Wenn der Film nicht erkannt wird, stellt sich die Frage, um was für einen Film es sich handelt. Es entsteht Unsicherheit. Zusätzlich entsteht Unsicherheit, da der Titel, wenn er nicht erkannt wird, nicht zur Steuerung der Kommunikation herangezogen werden kann. Beidem wird offenbar durch den deutlich verstärkten Rückgriff auf Genreangaben begegnet. Es wird nicht mehr der Film selbst erkannt und kommuniziert, sondern die Art des Films. Allerdings stellt sich die Frage, ob die Unterschiede bei der Verwendung der Genreangaben auf die Variation von Unsicherheit zurückzuführen ist.

In den Daten bietet sich diesbezüglich ein weiterer Vergleich an. In 150 der insgesamt 779 Ausschnittseinordnungen nennen die Probanden den Titel. In diesen Fällen kann plausiblerweise angenommen werden, die Probanden meinen, den Film erkannt zu haben. Diese Situation wäre also mit der Befragung vergleichbar. Abgesehen von den möglichen Fällen, in denen die Probanden den Titel geraten haben, müsste die zusätzliche Unsicherheit weitgehend eliminiert sein. Es dürften demnach kaum noch Unterschiede zwischen den Studien auftreten. Tatsächlich egalisieren sich die Unterschiede weitgehend: Zwar werden auch hier Genreangaben häufiger gebraucht als bei der bloßen Bezeichnung von Filmen in einer Kommunikationssituation. Das ließe sich aber durch Probanden erklären, die den Film nicht sicher erkennen, sondern raten, also noch unsicher sind. Ansonsten ist die Art der Bezeichnung, wenn der Titel genannt wird, relativ ähnlich, zum Teil sogar identisch. Die Ausnahme stellen die Verweise auf Personen dar. Die deutlich höhere Personennennung, wenn bei den Rezeptionsstudien der Titel genannt wird, erklärt sich durch Figuren als Wiedererkennungsfaktor. Vielfach erkennen die Probanden den Film anhand der Personen im Ausschnitt und benutzen dann sowohl den Titel als auch die Person zur Bezeichnung.

Dreht man die Argumentation um, so wird die Funktion der Genreeinordnung und -nennung noch deutlicher. In den Fällen, in denen die Probanden in den Rezeptions-

7 Dabei werden zur Schätzung der Varianz der Differenz weder die Varianzen der Prozentwerte beider Untersuchungen gepoolt noch die Varianz der Prozentwerte der Rezeptionsstudie an der Anzahl der Rezeptionen, sondern an der Anzahl der Probanden (also 124 statt 779) relativiert. Geht man zudem von ungünstigsten Fall, nämlich einer Verteilung von 50\% zu 50\% aus, ergibt sich ein $\mathrm{t}$-Wert von über 70 bei der Differenz von 25 Prozentpunkten und von über 140 bei den Differenzen von 50 Prozentpunkten, also $\mathrm{p}<0,001$. 
studien den Titel nicht nennen, haben sie die Sendung, aus dem der Ausschnitt stammt, wahrscheinlich nicht erkannt. In diesen Fällen wird das Genre zum dominierenden Bezeichnungselement. Es wird in 82 Prozent der Bezeichnungen verwendet und kommt damit in den meisten Bezeichnungen vor, in denen die Probanden überhaupt etwas meinen erkannt zu haben. Keine andere Elementgruppe kommt auf eine ähnliche Wichtigkeit: 57\% Gattungen, 23\% Inhalt, 10\% Personen, 12\% Produktion und 5\% Bewertung.

Ein Problem der vorangegangenen Untersuchungen besteht aber in der Feststellung der Unsicherheit. Bislang liegen den Vergleichen grundsätzliche Überlegungen zugrunde, welche Situation welche Unsicherheit bergen müsste. Ob die Untersuchten die angenommene Unsicherheit empfinden, ist nicht generell $\mathrm{zu}$ überprüfen, sondern nur durch individuelle Angaben.

\subsection{Genrebezeichnungen und die individuelle Sicherbeitseinschätzung}

Die Frage nach der Sicherheit der individuellen Einordnung des Ausschnitts war Bestandteil einiger Rezeptionsstudien. In diesen bezeichneten die Probanden 159 (23\%) Einordnungen als sehr sicher, 287 (43\%) als relativ sicher, 166 (24\%) als relativ unsicher und $71(10 \%)$ als sehr unsicher. Wenn sich die Probanden sehr unsicher sind, machen sie bei jeder achten Rezeption gar keinen Versuch, den Ausschnitt einzuordnen und zu bezeichnen.

\section{Grafik 1: Elemente nach Sicherbeitseinschätzung}

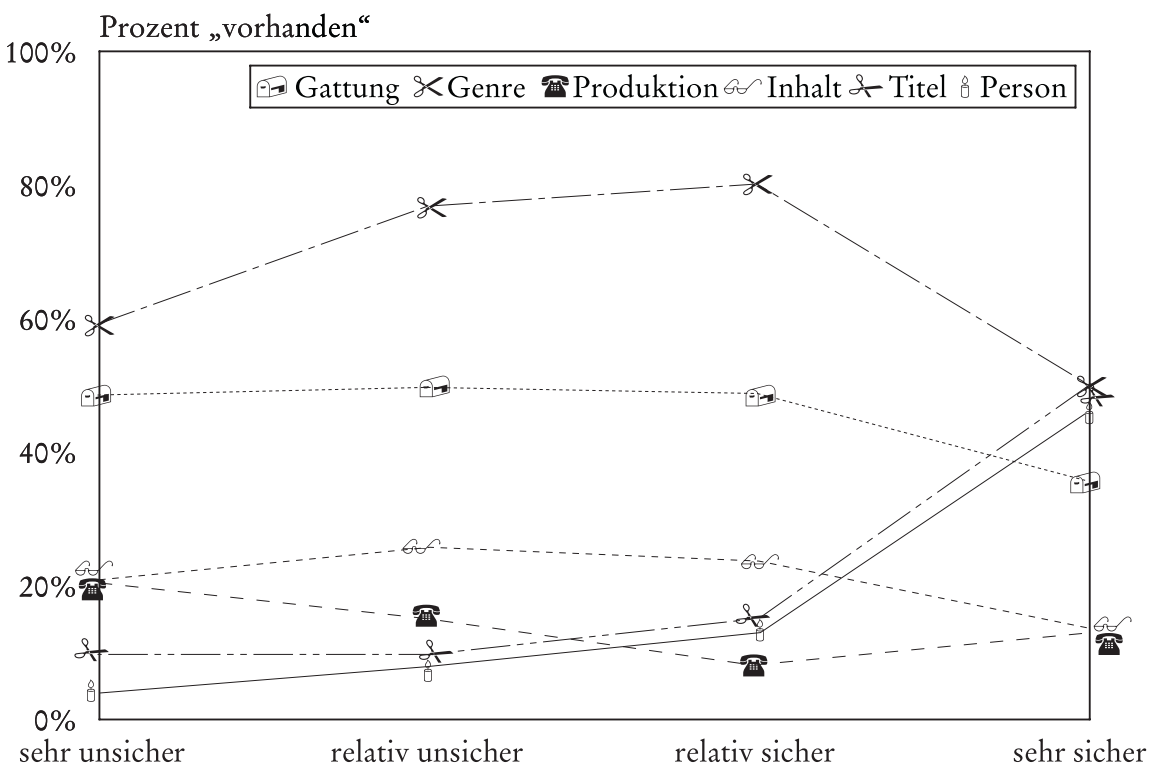


Genrenennungen sind in knapp sechs von zehn sehr unsicheren Beschreibungen enthalten. Relativ unsichere und relativ sichere kommen auf ca. acht von zehn Angaben, sehr sichere hingegen nur auf fünf von zehn. Gattungsangaben kommen in gut jeder zweiten unsicheren, relativ unsicheren und relativ sicheren Einordnung vor. Sind diese sehr sicher, sinkt der Anteil unter 40 Prozent. Inhaltsverweise liegen zwischen 21 und 26 Prozent, wenn die Einordnung nicht sehr sicher ist und gehen dann auf 13 zurück, wenn die Angaben sehr sicher sind. Das Vorkommen von Produktionsangaben vermindert sich von 20 Prozent bei sehr unsicher über 15 auf 8 bei relativ sicher und erhöht sich dann wieder auf 14 Prozent. Sind die Einordnungen unsicher oder relativ unsicher, finden sich jeweils 10 Prozent Titelverweise, werden sie relativ sicher, steigt der Anteil auf 15 Prozent und auf 50 Prozent, wenn die Angaben sehr sicher sind. Die Verteilung der Personenangaben ist ähnlich, sie liegt lediglich 2 bis 5 Prozentpunkte niedriger. ${ }^{8}$

Insgesamt liegt eine Zweiteilung der Effekte nahe. Wenn sich die Probanden bei ihrer Einordnung sehr sicher sind, stellen sich Effekte anders dar als unter Unsicherheitsbedingungen. Solange die Einordnung nicht sehr sicher ist, ergeben sich folgende Einflüsse: Mit zunehmender Sicherheit steigt der Anteil der verwendeten Genre- und Personenelemente. Je mehr Probanden meinen, Personen oder Genres zu erkennen, umso sicherer sind die Angaben im Durchschnitt. Ebenso verhält es sich mit dem Titel, allerdings findet der Anstieg erst zwischen relativ unsicher und relativ sicher statt. Inhaltsangaben verändern sich kaum nach Unsicherheit. Gattungsangaben nehmen tendenziell bei steigender Sicherheit ab, Produktionsangaben sogar deutlich.

Wenn man die Effekte in zwei Regressionsmodellen schätzt, bestätigt sich das eben angedeutete Bild. Im ersten Modell erklären die Bezeichnungselemente als unabhängige Variablen gut 6 Prozent der Varianz, der auf die drei Werte sehr unsicher, eher unsicher und eher sicher verkürzten Sicherheitsskala. Genreangaben korrelieren mit einem BetaWert von 0,16 signifikant positiv mit der Sicherheitseinschätzung. Je mehr Probanden

\section{Tabelle 3: Regressionsmodelle zur Sicherheitseinschätzung und Erkennung}

\begin{tabular}{|c|c|c|}
\hline Beta-Werte & Sicherheit der Einschätzunga & Erkennung des Ausschnittsb \\
\hline Genre & $0,16 * *$ & $-0,04$ \\
\hline Gattung & $-0,02$ & $-0,02$ \\
\hline Inhalt & 0,02 & $-0,06$ \\
\hline Titel & 0,09 & $0,27 * * *$ \\
\hline Person & $0,11 *$ & $0,25 * * *$ \\
\hline Bewertung & 0,08 & $-0,02$ \\
\hline Produktion & $-0,14 * \%$ & 0,00 \\
\hline Modell & $\mathrm{df} 7 / 505 ; \mathrm{R}^{2}=0,06 ; \mathrm{F}=4,3 * * *$ & $\mathrm{df} 7 / 664 ; \mathrm{R}^{2}=0,21 ; \mathrm{F}=26,3 * * *$ \\
\hline $\begin{array}{ll}* & \mathrm{p}>0,05 / * * * \\
\text { a Abhängige } \\
\text { sehr sicher }=\end{array}$ & $\begin{array}{l}\mathrm{p}<0,001 \\
\text { herheit, Skala: sehr unsicher }=1 / \text { relat }\end{array}$ & Insicher $=2$ /relativ sicher $=3 /$ \\
\hline
\end{tabular}

8 Vergleicht man jeweils die mittlere Häufigkeit des Auftretens jedes einzelnen Bezeichnungselementes zwischen den vier Sicherheitseinschätzungen, ergeben sich in Bezug auf die Bewertungen keine signifikanten Unterschiede. Infolge dessen wurde auf die Angabe der Bewertung zugunsten besserer Übersichtlichkeit verzichtet. 
Genrehinweise finden, umso sicherer wird die Einordnung eingeschätzt. Ebenso - wenn auch weniger deutlich - verhält es sich mit den Personenangaben (Beta =0,11). Produktionsangaben laufen den Effekten entgegen (Beta $=-0,14)$.

Das zweite Regressionsmodell begreift die Angabe, die Einordnung sei sehr sicher, als Erkennung des Ausschnitts. Stellt man diese Angabe in einem Regressionsmodell mit einer zweiwertigen abhängigen Variablen den drei Unsicherheitsbedingungen gegenüber, so wird gut 20 Prozent der Varianz erklärt. Sowohl Titel (Beta $=0,27$ ) als auch Personen (Beta $=0,25)$ verursachen den Unterschied, können also als die eigentlichen Erkennungsfaktoren angesehen werden. Genreangaben sind demgegenüber nicht mit der Einschätzung verbunden, den Film sicher erkannt zu haben.

\section{Zusammenfassung und Diskussion}

Die Studie geht dem Zusammenhang zwischen der Verwendung von Genrebezeichnungen und der Reduktion von Unsicherheit nach.

Im ersten Teil des Beitrags wurden vier Modellierungen vorgestellt, die die unsicherheitsreduzierende Funktion von Genres und Genrebezeichnungen verdeutlichen. Allen gemeinsam ist die Idee, dass Genres Erwartungen steuern. Wird ein Angebot einem Genre zugeordnet, werden einige Merkmale oder Phänomene relativ sicher erwartet, andere relativ sicher ausgeschlossen. In der kulturellen Modellierung resultiert die Reduktion von Unsicherheit aus der über Genres geschaffenen Anschlussfähigkeit zwischen einem konkreten Angebot und dem Gesamtangebot. Demgegenüber stehen die Anschlussfähigkeit des einzelnen Angebots an bereits erfolgreich vermarktete Angebote sowie die Publikumswünsche im Vordergrund der ökonomischen Modellierung. In der psychologischen Modellierung bieten Genrezuordnungen Anschlussmöglichkeit an bereits gemachte kognitive und emotionale Erfahrungen. Damit wird Unsicherheit in Selektions- und Rezeptionsprozessen reduziert. In der sozialen Modellierung dienen Genrebezeichnungen dazu, mittels Sicherung von Anschlusskommunikation Unsicherheit bei gemeinsamen Medienhandlungen, insbesondere der Kommunikation über Medienangebote, zu reduzieren.

Die vorgestellte Lesart der Fachliteratur wird im zweiten Teil auf das Publikum fokussiert und in zwei Hypothesen zusammengefasst: Zuschauer benutzen in der Kommunikation über Medienangebote verstärkt Genrebezeichnungen, wenn die Situation unsicher ist. Innerhalb derselben Situation steigt das individuelle Sicherheitsgefühl an, wenn auf Genrebezeichnungen zurückgegriffen werden kann. Die vorliegenden Daten bestätigen die Vermutungen. Genreangaben kommen in gut jeder vierten Bezeichnung vor, die Fernsehzuschauer bei der Kommunikation über fiktionale Fernsehangebote benutzen, sogar in jeder zweiten, wenn sie bei der Bezeichnung nicht den Titel verwenden. Diese Tatsache wird mit dem Versuch erklärt, Unsicherheit darüber zu reduzieren, ob der Gesprächspartner adäquat versteht, worüber man redet, was vor allem dann unsicher erscheint, wenn der Titel nicht zur Steuerung der Kommunikationssituation zur Verfügung steht. Wenn zu der benannten Unsicherheit über das Gelingen der Kommunikation noch die Unsicherheit darüber hinzukommt, um was für ein fiktionales Fernsehangebot es sich handelt, steigt die Häufigkeit der Genreangaben noch einmal deutlich an. So werden Genrebezeichnungen in fast drei Viertel der Angaben zu fiktionalen Fernsehangeboten benutzt, wenn die Zuschauer aus diesen lediglich einen Ausschnitt gesehen haben. Wird der Ausschnitt von den Zuschauern erkannt, nähert sich die Häufigkeit der Genreangaben der typischen Häufigkeit bei der Bezeichnung von gesehenen Sendungen an. Wenn der Ausschnitt demgegenüber nicht erkannt wird, 
steigt der Anteil der Genrebezeichnungen auf gut achtzig Prozent der Fälle. Demnach wird bei der Kommunikation über Fernsehangebote auf Genrebezeichnungen zurückgegriffen und zwar umso öfter, je mehr Unsicherheit die Situation birgt. Aber auch wenn man die individuelle Unsicherheit betrachtet, die jemand angibt, wenn er einen Ausschnitt einordnet, ergibt sich der erwartete Zusammenhang zwischen Unsicherheit und Genre: Die Zuschauer empfinden die Einordnung umso sicherer, je mehr genrerelevante Verweise sie finden, obgleich die Identifikation des Ausschnitts nicht mit diesen verbunden ist.

Genres im Allgemeinen sowie die Verwendung von Genrebezeichnungen im Besonderen erfüllen eine wichtige Funktion bei Handlungen mit oder in Bezug auf Medienangebote. Einzelne Genres sind mit bestimmten Merkmalen sowie individuellen Erfahrungen verbunden. Wird ein Angebot einem Genre zugeordnet, so wird vermutet, dass es auch diese Merkmale aufweist und ähnliche Erfahrungen hervorruft. Da diese Vermutung vielfach bestätigt wird, werden an Genres Erwartungen geknüpft. Durch die wiederkehrende Bestätigung der Genreerwartungen reduziert das individuelle Operieren mit Genres Unsicherheit, denn es macht konkrete kognitive und emotionale Erfahrungen durch den Anschluss an vorhergegangene vorherseh- und interpretierbar. Genrebezeichnungen machen die individuellen Erfahrungen kommunizierbar. In der Kommunikation werden die Bezeichnungen so abgeglichen, dass sie Verstehen und Anschlusskommunikation sichern, indem sie auf die Ähnlichkeit der individuellen Erfahrungen verweisen. So werden auf der sozialen Ebene Erwartungen gesteuert und Unsicherheit reduziert. Das wird zusätzlich dadurch begünstigt, dass sich bestimmte Genrebezeichnungen im kulturellen Diskurs als Standard durchgesetzt haben. Sie bieten die Möglichkeit, die Individualität einzelner Werke unter Bezug auf andere Werke desselben Genres zu begreifen und die kulturelle Kontinuität unter Bezug auf die Gesamtheit der Werke. So schaffen Genres und Genrebezeichnungen eine Verbindung einerseits zwischen individuellen und sozialen Erfahrungen mit Medienangeboten sowie andererseits zwischen individuellem und kulturellem Wissen über Medienangebote. Diese Verbindung nutzt man im ökonomischen Kontext, um Unsicherheit bei der Herstellung und Verbreitung von Film- und Fernsehangeboten zu reduzieren. Im Prozess der ökonomischen Spezialisierung wirken Genres zum Teil so stark, dass sich nach ihnen einzelne Marktsegmente mit speziellen Herstellungsverfahren und Vertriebswegen herausbilden.

\section{Literatur}

Altman, Rick (2000): Film /Genre. Bury St. Edmunds: British Film Institute Publishing.

Baumgartner, Elisabeth /Trauner, Wolfgang (1996): Das Museum als Erlebnisraum und seine Repräsentation in kognitiven Karten. In: Vitouch, P. /Tinchon, H. J. (Hrsg.), Cognitive Maps und Medien - Formen mentaler Repräsentation bei der Medienwahrnehmung. Frankfurt a. M. u. a.: Lang (Schriftenreihe zur Empirischen Medienforschung Band 1), S. 121 - 234.

Bawden, Liz-Anne (ed.) (1977): Buchers Enzyklopädie des Films. (Deutsche Ausgabe editiert von Wolfgang Tichy) Luzern, Frankfurt a. M.: Bucher.

Bordwell, David (1992): Kognition und Verstehen. Sehen und Vergessen in Mildred Pierce. In: montage /av 1, Nr. 1, S. 5 - 24.

Gehrau, Volker (1999): Genres und Gattungen als individuelle sowie sozial integrative Konstruktionen. In: Hasebrink, U. /Rössler, P. (Hrsg.), Publikumsbindungen - Medienrezeption zwischen Individualisierung und Integration. München: Fischer, S. 73 - 88.

Gehrau, Volker (2001): Fernsehgenres und Fernsehgattungen - Ansätze und Daten zur Rezeption, Klassifikation und Bezeichnung von Fernsehprogrammen. München: Fischer. 
Grodal, Torben (2000): Moving Pictures - A new theory of film genres, feelings, and cognition. Oxford: University Press.

Luhmann, Niklas (1987): Soziale Systeme - Grundriß einer allgemeinen Theorie. Frankfurt a. M.: Suhrkamp.

McConnell, Frank D. (1977): Leopards and history - The problem of film genre. In: Grant, B. K. (ed.), Film genre - Theory and criticism. Metuchen, London: Scarecrow, S. 7 - 15.

Minsky, Marvin (1975): A framework for representing knowledge. In: Winston, P. H. (ed.), The psychology of computer vision. New York: McGraw-Hill, S. $211-277$.

Neal, Steve (2000): Genre and Hollywod. London, New York: Routledge.

Neisser, Ulric (1979): Kognition und Wirklichkeit - Prinzipien und Implikationen der kognitiven Psychologie. Stuttgart: Klett-Cotta.

Ohler, Peter (1994): Kognitive Filmpsychologie - Verarbeitung und mentale Repräsentation narrativer Filme. Münster: MAkS Publikationen.

Rumelhart, David E. (1975): Notes on a schema for stories. In: Bobrow, D. G. /Collins, A. (eds.), Representation and understanding. New York: Academic Press, S. 211 - 236.

Rusch, Gebhard (1987): Kognition, Mediennutzung, Gattungen. SPIEL (6), 2, S. 227 - 272.

Schank, Roger C. /Abelson, Robert P. (1977): Scripts, plans, goals and understanding. Hillsdale: Erlbaum.

Scherer, Klaus R. (1998): Emotionsprozesse im Medienkontext: Forschungstraditionen und Zukunftsperspektiven. In: Medienpsychologie (10), 4, S. 276 - 293.

Schmidt, Siegfried J. (1987): Skizze einer konstruktivistischen Mediengattungstheorie. SPIEL (6), 2, S. 163 - 199.

Schwan, Stephan (1995): „Love or crime or something else?“ Schematische Wissensstrukturen und Filmrezeption. In: Rundfunk und Fernsehen (43), 1, S. 26 - 40.

Schweinitz, Jörg (1994): „Genre“ und lebendiges Genrebewußtsein - Geschichte eines Begriffs und Probleme seiner Konzeptualisierung in der Filmwissenschaft. In: montage /av (3), 2, S. 99-118.

Sobchack, Thomas (1977): Genre film - A classical experience. In: Grant, B. K. (ed.), Film genre Theory and criticism. Metuchen, London: Scarecrow, S. $39-52$.

Sobchack, Vivian (1982): Genre film - Myth, ritual, and sociodrama. In: Thomas, S. (ed.), Film /culture-Explorations of cinema in its social context. Metuchen, London: Scarecrow, S. 147 - 165.

Tudor, Andrew (1977): Genre. In: Grant, B. K. (ed.), Film genre-Theory and criticism. Metuchen, London: Scarecrow, S. 16 - 23.

Waldmann, Michael R. (1990): Schema und Gedächtnis. Heidelberg: Ansager.

\section{Anhang: Liste der bei den Rezeptionsexperimenten verwendeten Ausschnitte}

1 Mein Gott Willi, Komödie, BRD. Mann und Frau liegen im Ehebett, frühstücken und unterhalten sich dabei. Er sagt: „das große Verdienstkreuz“.

2 Julie Lescaut, Krimireihe, F. Eine schwarzhaarige Frau in Großaufnahme befindet sich in einem Raum, in dem im Hintergrund eine zweite Frau bügelt.

3 Länder - Menschen - Abenteuer, Bericht N3, BRD 1995. Blick auf den zentralen Platz eines Urwalddorfes. 2 dunkelhäutige Männer kommen mit Lanzen und Bündeln über den Platz gelaufen. Off-Stimme: „tauchen überraschend wieder auf“.

4 Bullit, Aktion, USA 1968. Mann geht wachsam um sich sehend durch einen Kellergang, von einem vergitterten Verschlag heraus gefilmt.

5 Bericht, $\mathrm{n}$ tv - Nachrichten, BRD 1995. Nachrichtensprecherin in rotem Sakko steht, in Handmikro sprechend, vor Plenarsaal mit aufgestellten Fahnen. Sie ist halbtotal zu sehen und sagt: „Minister und Vertreter“.

6 Scarlett 3, Ausstattungsfilm, BRD / I 1994. Eingangshalle einer Villa, farbiger Butler in weißem Dress öffnet Tür, rot gekleidete Frau kommt herein.

7 Madonna: Rain, Musikvideo, USA 1993. Blick in Filmstudio, erst hell, dann dunkel werdend; in weiß gekleidete Frau vor dem Großbild eines bewölkten Himmels posiert, Schnitt, weiß gekleidete Frau fliegt vor wolkigem Himmel.

8 Der Räuber mit der sanften Hand, Thriller, BRD 1995, RTL. Blick auf eine Terrasse an ei- 
nem Ferienort, 2 junge Männer mit offenen Hemden kommen an und eine Männerstimme sagt: „Hallo Bobby“.

9 Die Straßen von Berlin, Krimi-Reihe BRD 1995. Mann wird mit Revolver bedroht. Er spricht verängstigt: „Mann, wir sind doch“ und zieht seinen Pullover hoch.

10 Mann muss nicht sein, Comedy - Serie, USA. Typisch amerikanisch aussehende Frau mit schwarzen Locken und rotem Pulli ist in Großaufnahme zu sehen und sagt: „Nebenbei das sind meine zwei“.

11 Die Legende von O. B. Taggert, Western, USA 1994. Mann mit Hut am Zaun einer Ranch, hinten reitet ein Mann in Richtung Horizont, Überblende in einen Mondaufgang über dem Horizont.

12 Deadly Revenge, USA 1991, Action-Thriller. Hand greift in Schublade mit Geld und Pistole.

13 Asterix \& Kleopatra, Abenteuer / Zeichentrick, F / B 1968. Asterix und Obelix im Schneesturm.

14 Godzilla und die Riesenkäfer, Fantasy, J 1971. 3-köpfiger Drache speit Feuer.

15 Emanuelle in Tibet, Erotik, F 1993. Mann küsst nackter Frau den Po im Himmelbett liegend.

16a Deadly Revenge, Action-Thriller, USA 1991. Mann B mit Messer wartet / Schnitt / Mann A schreitet durch einen Laden.

16b Deadly Revenge, Action-Thriller, USA 1991. Mann A geht auf einen Laden zu / Schnitt / Mann A schreitet durch den Laden.

17a Ausbruch der 28, Kriegs-Film, USA 1969. Gesicht A mit Uniformmütze / Schnitt / Gesicht B.

17b Ausbruch der 28, Kriegs-Film, USA 1969. Gesicht B.

18a Herkules erobert Atlantis, Abenteuer-Film, I / F 1961. Berittene Römer: „Da ist er“ / Schnitt / Fluchtszene auf langem Weg.

18b Loriot auf Sofa: „Meine Damen und Herren“ / Schnitt / Herkules erobert Atlantis, Abenteuer-Film, I / F 1961. Fluchtszene.

19a Loriot: Von Menschen und Möpsen, Komödie, BRD 1989. Gezeichnete Hunde: „2 Namen werden wir uns künftig merken müssen, Bubbel und Lohrmann“.

19b Loriot: Von Menschen und Möpsen, Komödie, BRD 1989. Loriot auf Sofa: „Meine Damen und Herren“. / Schnitt / gezeichnete Hunde: „2 Namen ... Bubbel und Lohrmann“.

20 Basic Instinct, Erotik-Thriller, USA 1991. Leicht bekleidete Frau steigt aus Auto und läuft durch Regen zu einem Haus.

21 Bananas, Komödie, USA 1971. Mann (W. Allen) greift in seiner Küche nach gefrorenem Spinat, der ihm immer wieder entgleitet.

22 Bartholomäusnacht, Historien-Drama, F 1994. Reiter kommt zu Rittern mit Bewaffnung, die an einem Waldrand warten.

23 Super Mario Bros., Komödie, USA 1993. 2 Männer fliegen durch eine belebte Passage, viele Menschen sehen zu.

24 Basic Instinct, Erotik-Thriller, USA 1991. Paar liegt auf Fußboden, sie fragt ihn: „Wer ist sie gewesen?"

25a Die unglaubliche Reise in einem verrückten Raumschiff, Komödie, USA 1982. Mann flüchtet an einer Mauer entlang, an der ein Scheinwerferkegel kreist. Schlagermusik.

25b Die unglaubliche Reise in einem verrückten Raumschiff, Komödie, USA 1982. Mann flüchtet an einer Mauer entlang, an der ein Scheinwerferkegel kreist und trifft auf Schlagersänger. Schlagermusik.

26a Verschwörung der Frauen, Drama, UK 1988. Gesicht eines älteren Mannes taucht aus dem Wasser einer Zinkwanne auf und sagt: „Nancy hat mich gewaschen“.

26b Verschwörung der Frauen, Drama, UK 1988. Hand hält Gesicht unter Wasser einer Zinkwanne, wird weggezogen und der Kopf taucht langsam auf.

27a Adams Family, Grusel-Komödie, USA 1991. Das Eiskalte Händchen läuft, von der Kamera verfolgt, einen Schlossflur entlang.

27b Adams Family, Grusel-Komödie, USA 1991. Die Kamera fährt kurz über dem Boden einen Schlossflur entlang. 
28a Monty Python's wunderbare Welt der Schwerkraft, Komödie, UK 1971. Soldaten liegen am Boden, lachen leise und sterben.

28b Monty Python's wunderbare Welt der Schwerkraft, Komödie, UK 1971. Soldaten biegen sich vor Lachen und fallen vor Lachen um.

29a Basic Instinct, Erotik-Thriller, USA 1991. Nackte Frau stützt sich, den Oberkörper aufrecht haltend, auf das Bett auf dem sie liegt.

29b Basic Instinct, Erotik-Thriller, USA 1991. Nackte Frau stützt sich, den Oberkörper aufrecht haltend, auf das Bett auf dem sie liegt und zieht ein Seil aus dem Bettzeug hervor. 